\title{
Mandibular Permanent Canine with Two Root Canals, Two Roots: Case Report, Al-Medina, Saudi Arabia
}

\author{
Reem Adel Abdelgawad ${ }^{1 *}$, Maha Zarea Aljohani² ${ }^{2}$ and Nevine M Taymour ${ }^{3}$ \\ ${ }^{1}$ Assistant Professor, Restorative Dental Sciences Department, Saudi Arabia \\ ${ }^{2}$ Senior Dental Student, Saudi Arabia \\ ${ }^{3}$ Professor, Restorative Dental Sciences Department, Saudi Arabia
}

*Corresponding author: Reem Adel Abdelgawad, Assistant Professor, Restorative Dental Sciences Department, Saudi Arabia

\section{ARTICLE INFO}

Received: 㓞 July 15, 2019

Published: 㹃 July 24, 2019

Citation: Reem Adel Abdelgawad, Maha Zarea Aljohani, Nevine M Taymour. Mandibular Permanent Canine with Two Root Canals, Two Roots: Case Report, Al-Medina, Saudi Arabia. Biomed J Sci \& Tech Res 20(1)-2019. BJSTR. MS.ID.003385.

\begin{abstract}
The knowledge of different diverse human teeth roots and root canals morphology and the ability to read its "road map" is right road for the success of root canal treatment. Double rooted anterior teeth are rarely reported. This case report aim was to represent management of a case has mandibular canine with two root canals within two separate roots. Endodontic treatment of tooth \#33 with such morphology was performed in a 41-year-old woman. Therefore, clinicians must always suspect anatomical varieties and do not ignore anatomical variations even if recorded low prevalence, use all the available tools to diagnose, carefully examine the initial radiograph which is extremely important for these cases management.
\end{abstract}

Keywords: Anatomic Variation; Root Canal Morphology; Mandibular Canine; Root Canal Treatment

\section{Introduction}

Natural dentition retaining in function and esthetics is the golden goals of endodontic treatment, achievement of such goals depends on many steps starting from proper case selection and treatment plan up-to final restoration [1]. Root canals preparation regarded as the most critical step due to its direct effect on subsequent steps in the procedure (Ova A Peters) [2]. Failure due to incomplete root canal treatment may cause postoperative disease, pain, and complications [3]. One of the main of endodontic treatment failure causes is the inability of the clinician to detect extra canals because of the unexpected anatomy of some of the root canal systems [4]. For this reason, endodontists have to owe the awareness of the possible anatomical variations of root canals morphology of different teeth, adequacy of careful examination of the initial radiograph and should have the expertise to use modern magnification devices that help them achieve good success rate [5]. Most of mandibular canines show type I root canals (one root, one root canal), about 15\% might have two canals and a fewer number reported two distinct roots [6,7]. Anatomical variations associated with permanent mandibular canines have been reported by several investigators in Saudi Arabia [8,9]. Without performing extensive imaging examinations, it is difficult to precisely determine by clinical means whether a mandibular canine presents only one root with two independent apical foramens or actually has two separate roots. The present case report presents the endodontic management of a mandibular canine has two root canals within two separate roots. There is no case report done before in alMedina for endodontic treatment of a mandibular canine with two roots and two root canals. The following clinical report describes the non-surgical endodontic treatment of a mandibular canine with two roots and two root canals.

\section{Case Report}

A 41- year-old medically fit female visited the Dental Clinics and, Hospital of TIBAH University, Al-Medina, Saudi Arabia suffering from severe pain related to her lower left anterior region. The chief complaint was " sharp lingering pain upon cold stimulus in left mandibular canine and lasts for one minute even". Clinical examination revealed a mandibular left canine with deep caries in the buccocervical region of the crown (Figure 1). Periodontal examination showed; no tenderness to percussion, no mobility or periodontal pocket. Vitality tests of the involved tooth showed; 
increased reaction at Electrical Pulp Test and lingering pain to cold. Pre-operative periapical radiographs in different angulations revealed deep cervical caries approximated the pulp two apparent roots (Figure 2).

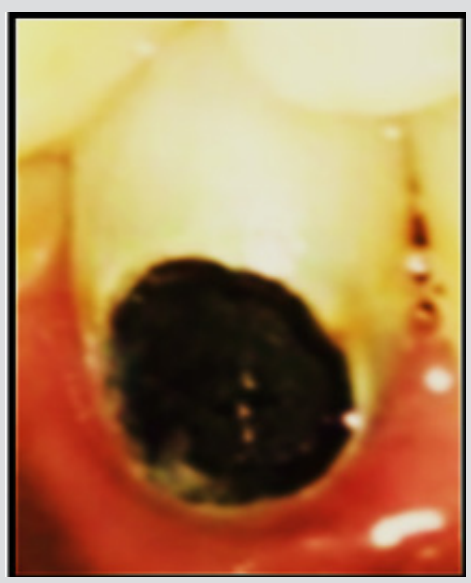

Figure 1: Photograph of mandibular canine showing deep caries in the bucco-cervical region.

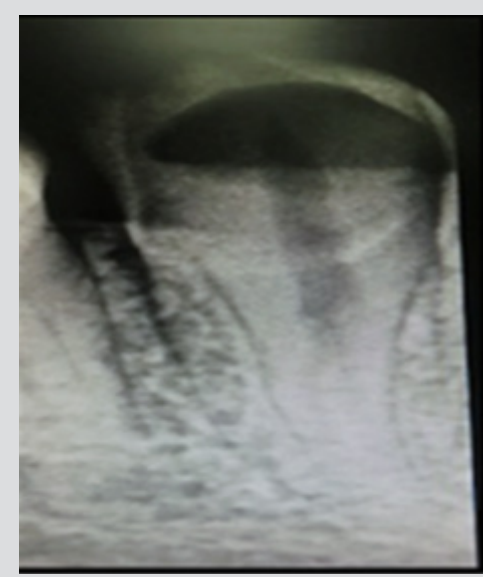

Figure 2: Preoperative radiograph of mandibular canine revealed two separated roots.

Clinical diagnosis showed symptomatic irreversible pulpitis with normal periapical tissues of left mandibular canine and the case was scheduled for nonsurgical root canal treatment of the involved tooth. After the local anesthetic administration (Lidocaine HCL 2\% with Epinephrine 1:100,000) and under rubber dam isolation, caries was removed from buccal surface then sealed using glass ionomer cement (Fuji, GC, Tokyo, Japan)and the pulp chamber was accessed from lingual aspect using round and Endo-Z bur (Dentsply Maillefer, Switzerland) (Figure 3). To enhance the accessibility to the canals, the access opening was modified including more the lingual surface. Two main orifices were found: one lingual and one buccal. Canals were negotiated with \#10 and \#15 k files (Dentsply, Maillefer, Ballaigues, Switzerland). Coronal flaring was carried out using \# 3, 2 and1 Gates Glidden drills (Dentsply, Maillefer, Switzerland). Working lengths were established using apex locator (Root ZX; J Morita Co, Kyoto, Japan) and verified by periapical radiograph (Figure 4 ).

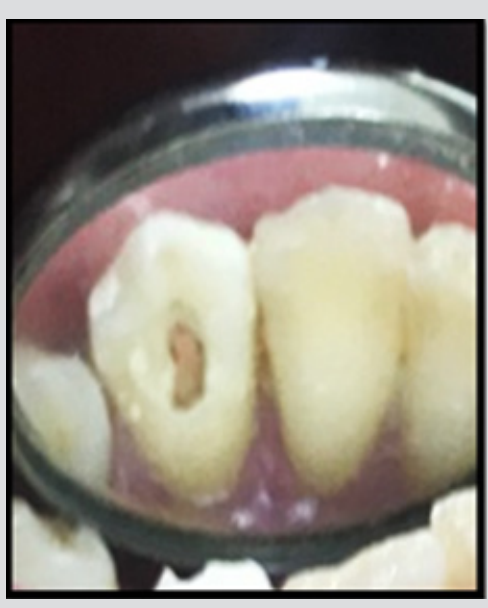

Figure 3: Access cavity showing separate orifices of two separate canals.

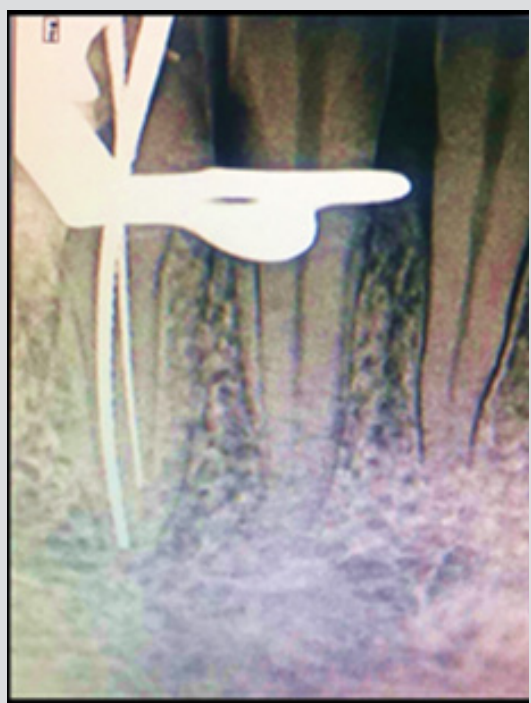

Figure 4: Working length measurement radiograph with two K-files in two separate roots.

Both the buccal and lingual canals were instrumented to an apical size \#40 in baccal canal and \#35 in lingual canal followed by step back instrumentation in both canals up to \# $60 \mathrm{k}$ file. Root canals irrigation was done using $5 \mathrm{ml}$ of $3 \% \mathrm{NaOCl}$ with each file use and final rinse using $5 \mathrm{ml}$ of saline. After chemo-mechanical preparation, root canals were dried using sterile paper points, and then obturated with cold Lateral Condensation Filling Technique using gutta percha 2\% (Dentsply-Maillefer, Ballaigues, Switzerland) and SealApex (Kerr Manufacturing Co) root canal sealer. Master gutta percha cone \# 40 in buccal canal, \#35 in lingaual canal were inserted into canals, tug back was confirmed and evaluated radiographically (Figure 5). Inserting sealer coated spreader size \#25 (Mani Inc., Tochigi, Japan) next to the master cone, $1 \mathrm{~mm}$ shorter than the working length followed by insertion of accessory gutta-percha cones of respective size in the provided space by the spreader. This sequence was repeated till the spreader penetration was not more than 1-2 mm from the cemento-enamel junction. A plugger was heated and used to remove gutta-percha excess with no further vertical compaction. The access preparation was there after restored 
with glass ionomer cement, post-operative radiographic evaluation and CBCT (Cone Beam CT) of the obturation revealed proper length, density, and lateral canals seal (Figures 6-8). One week later, the patient clinical examination showed no symptoms and the tooth restored with composite resin final restoration (Table 1).

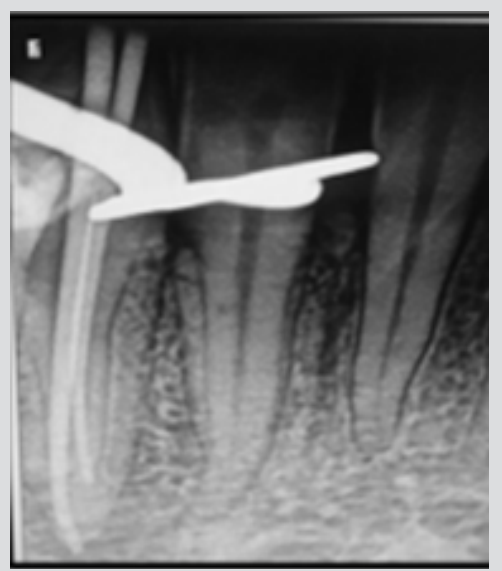

Figure 5: Master cone adjustment in both canals.

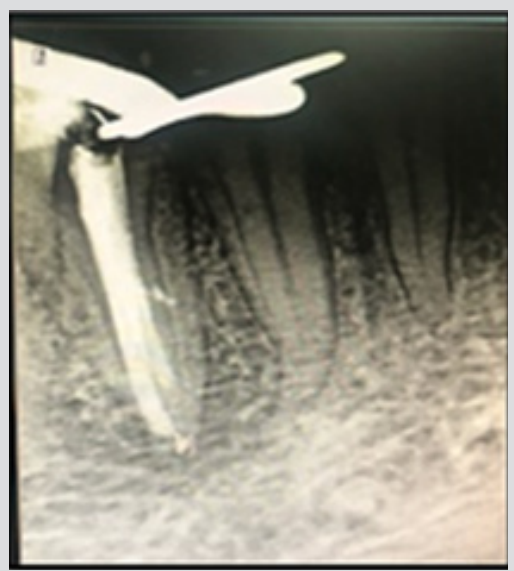

Figure 6: Obturation of both canals, lateral canals seal.

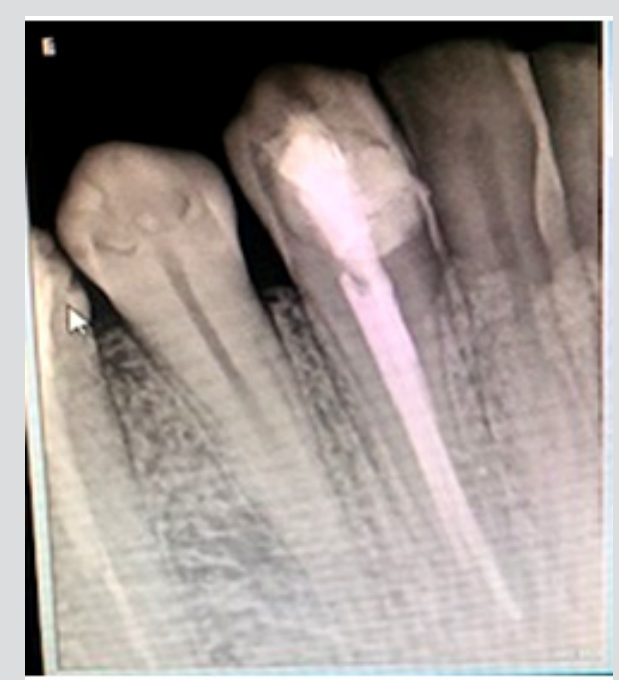

Figure 7: Root canals obturation and coronal restoration.

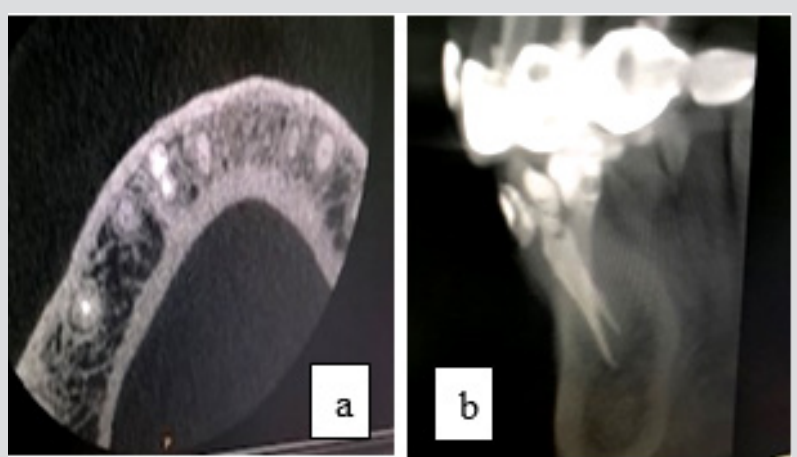

Figure 8 a \& b: CBCT images of the obturation of both root canals.

\section{Discussion}

Success of root canal treatment mainly depends on proper diagnosis, case selection, and treatment planning which achieved by integrating clinical and radiographic findings for a better clinical outcome. Apart from effectively completing the clinical part of the examination process, the radiographic aspect represents a core element in diagnosis and management of endodontic disease. Careful examination of the initial radiograph is extremely important for identification of multiple roots, root canals, and anatomical variations. Without performing extensive imaging examinations, it is difficult to precisely determine by clinical means whether a mandibular canine presents only one root with two independent apical foramens or actually has two separate roots. It is most common for a mandibular canine to present with (Type 1) classification that is single root canal extending from the chamber of the pulp to the root apex. However, variable root canal morphologies can occur such as two root canals within a single root [1-9] and in some complex cases, three root canals within one or two roots have been reported [10-12].

Cases have mandibular canines with two root canals within two roots were reported $[13,14]$. The present case report presented; a Saudi female who required a non-surgical root canal treatment on her permanent mandibular canine have two roots and two root canals. A pre-operative periapical radiograph was taken with various horizontal angulations. The initial radiograph revealed anatomical variations from the normal in relation to the mandibular canine (\#43) which presented with two roots and two root canals; namely one buccal and lingual, respectively. The two canals have two separate buccal and lingual orifices within pulp chamber till the root apex of the buccal and lingual roots respectively. In this present case, an adequately prepared access cavity with more lingual extension) helped to clearly visualize the pulpal floor in which buccal and lingual orifices were detected and accessed appropriately forming an access preparation with an ovoid outline bucco-lingually. Periapical radiographs at different horizontal angulations formed the core milestone element in this presented case report, as it confirmed the occurrence of variations in the root anatomy in relation to \# 43 . 
Properly angulated periapical radiographs taken at more than one angle allowed for separation of buccal and lingual roots and limited their superimposition $[15,16]$. Periapical radiographs with different horizontal angulations for both working length determination and master cone placement were further performed and were found to support the previous initial results; presence of buccal and lingual roots for \# 43 with buccal and lingual canals in each root respectively. Buccal root and buccal canal was longer than its component lingual root and lingual canal. More advanced tool for radiographic investigation such as Cone-Beam Computed Tomography (CBCT) have been recently introduced to give accurate and specific information about root canal variations [17]. However, in the present case report, the anatomical variation was obtained by using periapical radiographs with various horizontal angulations. It must thus be emphasized that a sound knowledge of different teeth roots anatomy and morphology is mandatory before attempting any root canal diagnosis and treatment planning as well as careful examination of the initial radiograph.

Failure to do so may lead to an endodontic mishap due to the superimposition of any existing roots and root canals, and lack of cleaning and shaping of the obscured ones, allowing them to act as an existing source of infection that may presumably end up in a root canal failure. Recently, magnification tools and devices such as fiber optics, magnification loupes, and the Dental operating Microscope are becoming more popular in the endodontic practice, and are used by many of the endodontists to help detect the presence of extra roots and extra canals by increasing illumination and magnification [18]. It is worth noting that some researchers attributed variations in root canal anatomy from the most common types to certain factors, such as genetics, sex, race, as well as to some extent the researches' judgment and experience [8].

\section{Conclusion}

In spite of rare occurrence of mandibular canines have two separate roots recorded by the literature, clinicians should always suspect the occurrence of variations in teeth they are treating and should never assume that root canal morphology is simple. Always a careful radiographic interpretation with different angulations must be done. The diagnosis and meticulous exploration of the variable anatomy of a mandibular canine allow for good the planning of management protocol and success of root canals treatment.

\section{References}

1. Pineda F, Kuttler Y(1972) Mesiodistal and buccolingual roentgenographic investigation of 7,275 root canals. Oral Surg Oral Med Oral Pathol 33: 101-110.

2. Peters OA (2004) Current challenges and concepts in the preparation of root canal systems. Journal of Endodontics 8 (30): 559-567.

3. Sjogren U, Hagglund B, Sundqvist G, Wing K (1990) Factors affecting the long-term results of endodontic treatment. J Endod 16: 498-504.

4. Friedman S (2002) Prognosis of initial endodontic therapy. Endod Topics 2: 59-88.

5. Vertucci FJ (1984) Root canal anatomy of the human permanent teeth. Oral Surg Oral Med Oral Pathol 58: 589-99.

6. Green D (1973) Double canals in single roots. Oral Surg Oral Med Oral Pathol 35: 689-96.

7. Sert S, Bayirli GS (2004) Evaluation of the root canal configurations of the mandibular and maxillary permanent teeth by gender in the Turkish population. J Endod 30: 391-398.

8. Caliskan MK, Pehlivan Y, Sepetcioglu F, Turkun M, Tuncer SS (1995) Root canal morphology of human permanent teeth in a Turkish population. J Endod 21: 200-204.

9. Arora V, Nikhil V, Gupta J (2013) Mandibular canine with two root canals - An unusual case report. Int J Stomatol Res 2: 1-4.

10. Heling I, Gottlieb Dadon I, Chandler N (1995) Mandibular canine with two roots and three root canals. Dent Traumatol 11: 301-302.

11. Holtzman L (1997) Root canal treatment of a mandibular canine with three root canals. Case report. Int Endod J 30: 291-293.

12. Orguneser A, Kartal N (1998) Three canals and two foramina in a mandibular canine. J Endod 24: 444-445.

13. Rahmatulla M, Wyne AH (1993) Bifid roots in a mandibular canine: Report of an unusual case. Saudi Dent J 5: 77-78

14. Balto HA, Al Wakeel M (2007) Mandibular canine with two root canals Case report. Egypt Dent J 53: 2535-2538.

15. Slowey RR (1974) Radiographic aids in the detection of extra root canals. Oral Surg Oral Med Oral Pathol 37: 762-772.

16. Fava LR, Dummer PM (1997) Periapical radiographic techniques during endodontic diagnosis and treatment. Int Endod J 30: 250-261.

17. Patel S (2009) New dimensions in endodontic imaging: Part 2. Cone beam computed tomography. Int Endod J 42: 463-475.

18. Carr GB (1992) Microscopes in endodontics. J Calif Dent Assoc 20: 5561.

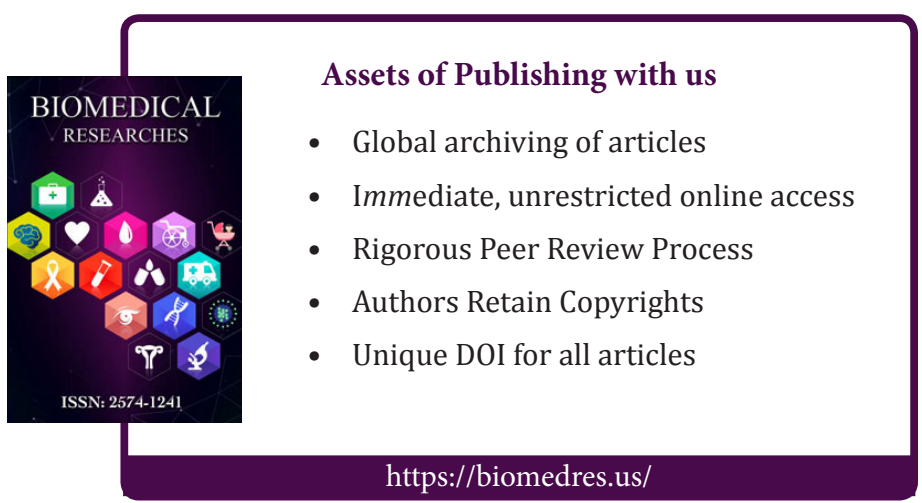

